Ann. Zootech., 1982, 31 (2), 111-130

\title{
Etude de la prévision de la digestibilité des fourrages par une méthode enzymatique
}

\author{
Jocelyne AUFRERE \\ I.N.R.A., Laboratoire des Aliments, \\ Centre de Recherches zootechniques et vétérinaires de Clermont-Ferrand \\ Theix, F 63110 Beaumont
}

\section{Résumé}

La méthode proposée comporte deux étapes : un pré-traitement avec de la pepsine dans l'acide chlorhydrique $1 \mathrm{~N}, 24$ heures à $40^{\circ}$, suivi d'un traitement par une cellulase "Onozuka R $10 », 24$ heures à $40^{\circ}$.

Nous avons vérifié que :

- la liaison entre la digestibilité in vivo et celle par la pepsine-cellulase était améliorée par la présence de pepsine $\left(S_{R}=0,036\right.$ avec pepsine contre $S_{R}=0,041$ sans pepsine) mais ne l'était pas par la concentration en acide (entre $0,1 \mathrm{~N}$ et $1 \mathrm{~N}$ ); toutefois avec la valeur de $1 \mathrm{~N}$ choisie, la valeur de la digestibilité par la pepsine-cellulase se rapproche de celle de la digestibilité in vivo;

- la précision de la prévision n'était pas meilleure si la température d'incubation augmentait de $40^{\circ}$ à $50^{\circ}$, ou si le temps d'incubation passait de 24 heures à 48 heures, que ce soit pour le pré-traitement pepsine dans l'acide chlorhydrique ou le traitement par la cellulase ;

- la cellulase «Onozuka R 10 » utilisée était particulièrement active et pouvait être employée à des doses $(50 \mathrm{mg} / 500 \mathrm{mg}$ de fourrage) plus faibles que celles préconisées dans la bibliographie. La précision de la prévision avec ce type d'enzyme était voisine de celle des autres cellulases étudiées : "Celluclast» et "Sigma ».

Appliquée à 154 échantillons de fourrages verts, de foins et d'ensilages cette méthode a permis de prévoir leur digestibilité à l'aide d'une seule équation de régression (sauf pour les trèfles violets) avec une bonne précision $\left(S_{\mathrm{n}}= \pm 0,023\right)$.

\section{Introduction}

La digestibilité des fourrages varie selon l'espèce végétale et pour une espèce donnée selon le stade de végétation, l'âge ou le numéro du cycle. L'essentiel des résultats obtenus à l'I.N.R.A. pour les fourrages verts a été présenté dans les «Tableaux de la valeur alimentaire des fourrages » (DEMARQuilly \& WEISS, 1970) et dans les tableaux de la valeur nutritive des aliments (Demarquilly, Andrieu \& SaUVANT, 1978). Ces résultats ont été complétés par des équations permettant de prévoir la valeur nutritive et l'ingestibilité à partir de l'âge du fourrage et de sa composition 


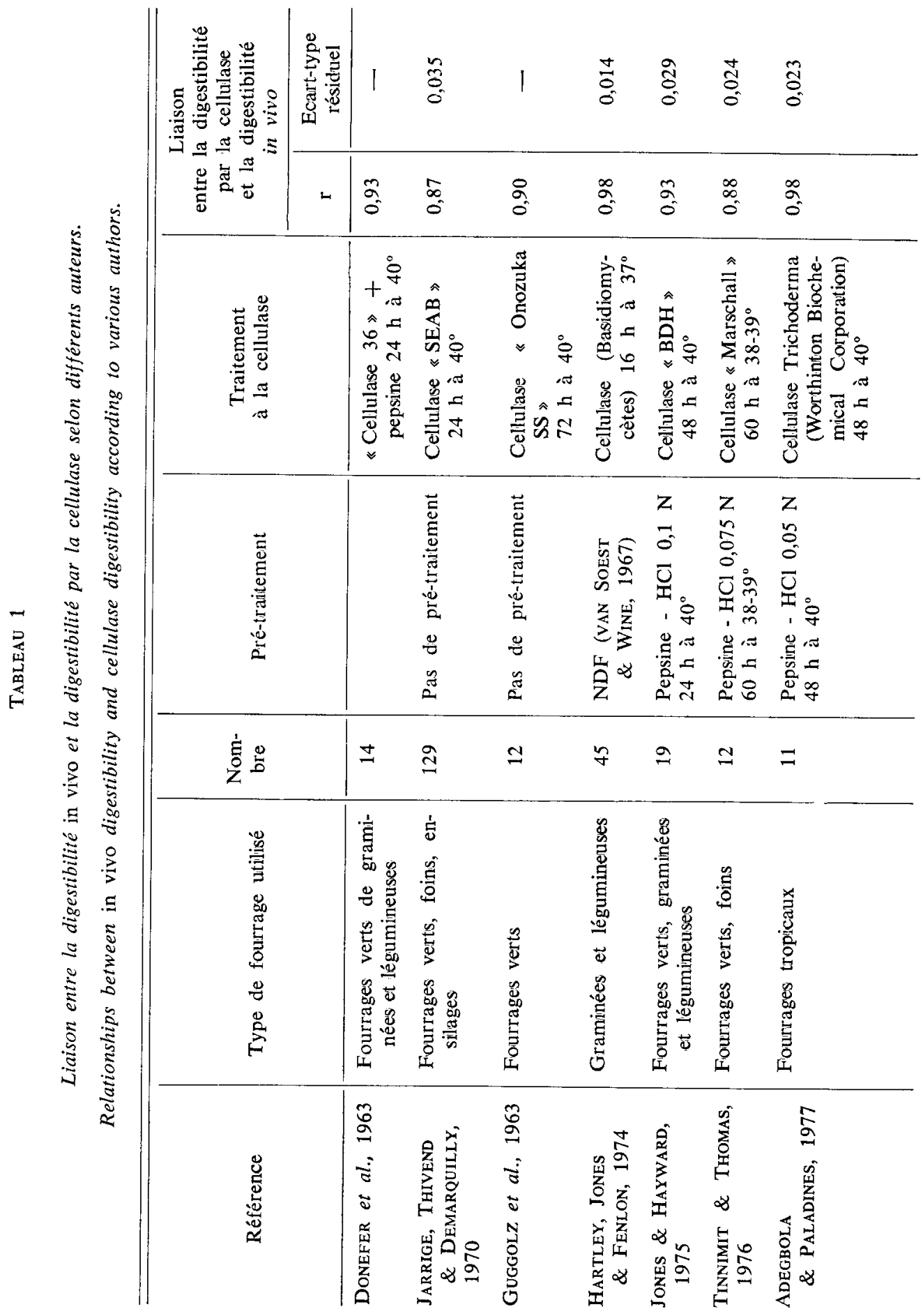




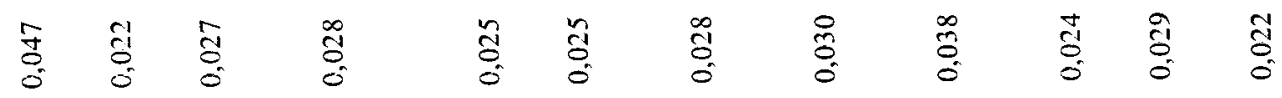

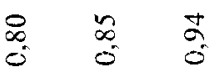
ô
$\vec{\sigma} \quad \vec{a}$
$\underset{\infty}{\infty}$
$\vec{n} \quad 1 \quad \hat{\sigma}$

$\stackrel{\Xi}{\Xi}$

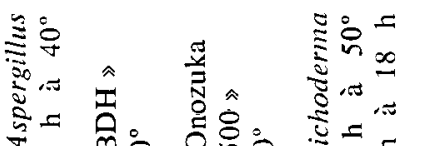

+
$\therefore$

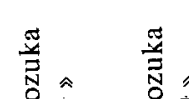

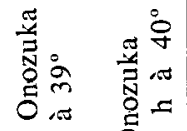

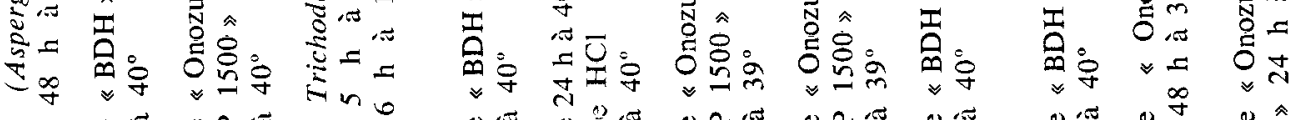

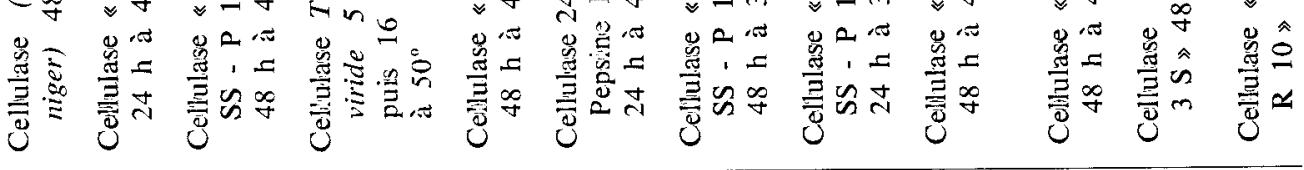

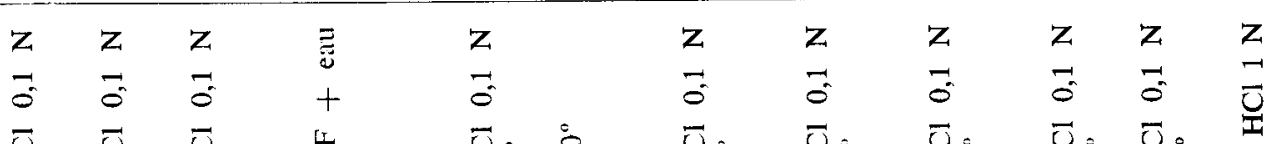

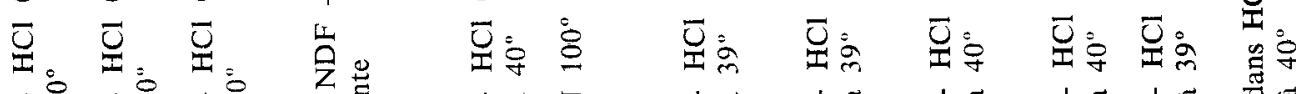

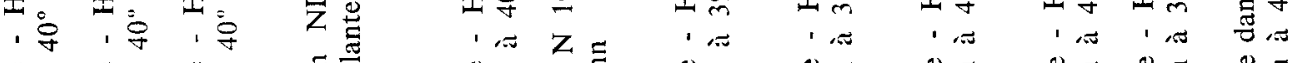

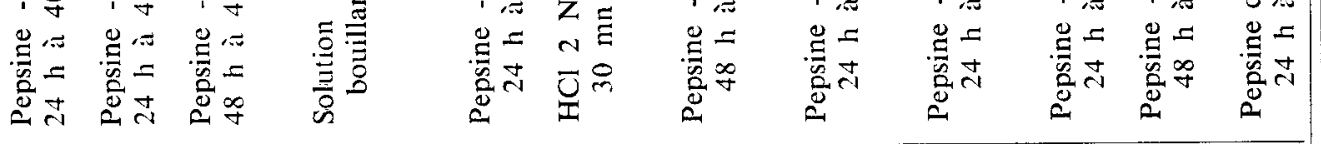

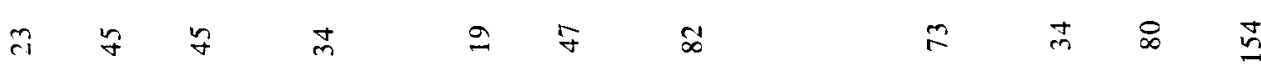

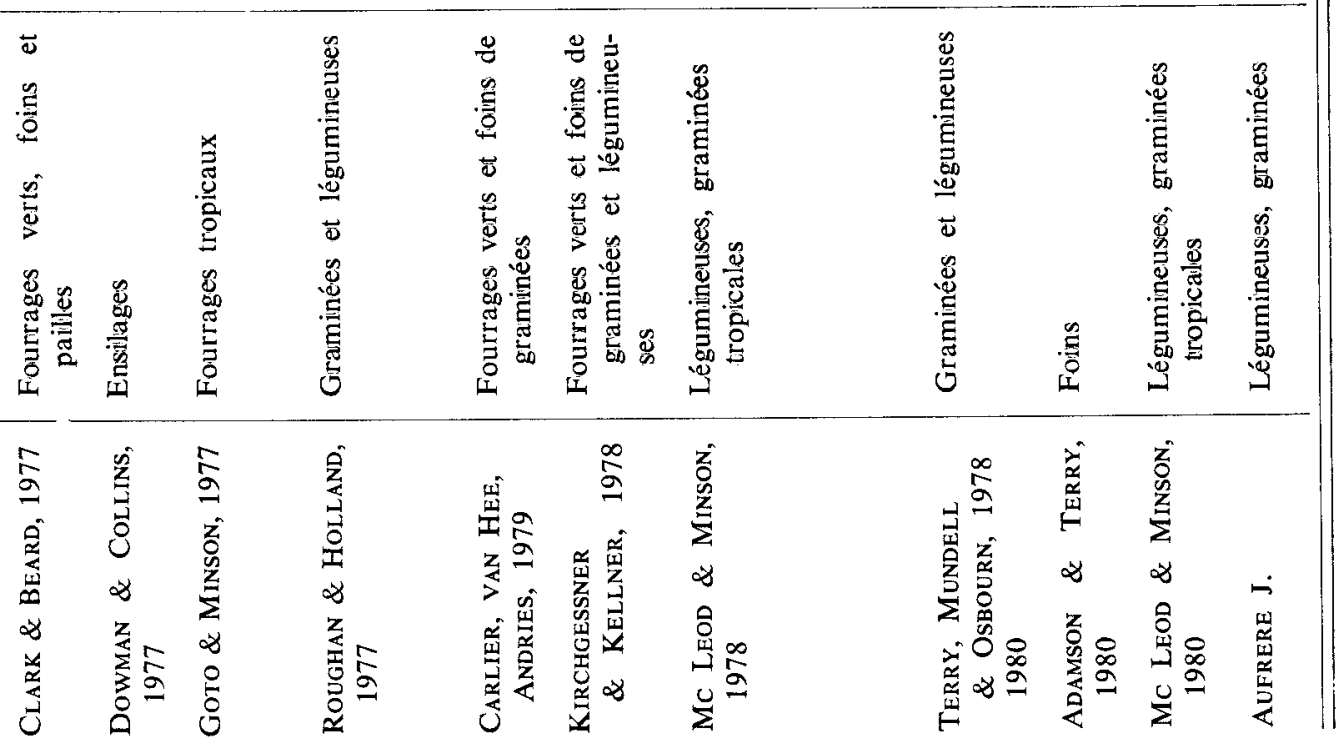


chimique classique (Andrieu, Demarquilly \& Wegat-Litre, 1981). Ces équations ne sappliquent cependant pas à tous les fourrages, notamment à ceux des prairies à flore complexe : mélanges semés et prairies naturelles. Il faut alors avoir recours à d'autres méthodes de laboratoire.

Parmi les différentes méthodes de laboratoire existantes (DEmarquilly \& JARRIGE, 1981), i! spparaît que les méthodes microbiologiques mettant en œuvre les microbes du rumen sont incontestablement les plus précises mais elles sont hors de portée des laboratoires de série puisqu'elles nécessitent l'entretien d'animaux porteurs de fistules du rumen.

Pour pallier cet inconvénient, depuis une quinzaine d'années, de nombreux auteurs ont testé des préparations cellulasiques pour prévoir la digestibilité in vivo (tabl. 1). JARrige \& Thivend (1969) utilisent une cellulase fongique seule (SEAB) et obtiennent une bonne liaison entre la digestibilité par la cellulase et celle in vivo sur les 129 échantillons testés. Cependant un pré-traitement semble particulièrement efficace pour accroître la sensibilité des parois à la cellulase : solution au détergent neutre (Hartley, Jones \& Fenlon, 1974 ; MC Queen \& Van Soest, 1975 ; Rexen, 1977 ; Roughan \& Holland, 1977 ; Israelsen, Rexen, Vestergaard Thomsen, 1978), eau bouillante et $\mathrm{Na}_{2} \mathrm{SO}_{3}$ (ABE, HorII \& KAMEOKA, 1972), pepsine dans de l'acide chlorhydrique (DonefER et al., 1963 ; Jones \& Hayward, 1975). Depuis de nombreux auteurs (Adegbola \& Paladines, 1977 ; Goto \& Minson, 1977 ; Terry, Mundell \& Osbourn, 1978) utilisent ce dernier pré-traitement.

La méthode proposée par Jones \& HaYward (1975) a été l'une des plus utilisée pour prévoir la digestibilité des fourrages. Elle comprend deux étapes : un pré-traitement par la pepsine dans de l'acide chlorhydrique dilué $(0,1 \mathrm{~N})$ pendant 24 heures suivi d'un traitement par la cellulase pendant 48 heures. Cette méthode a servi de base à notre travail.

Notre but était d'obtenir :

- une valeur de digestibilité par la pepsine-cellulase voisine de la digestibilité in vivo de la matière organique : nous avons essayé pour cela de définir des conditions optimales pour le pré-traitement d'une part et l'attaque par la cellulase d'autre part, ainsi que le type de cellulase à utiliser ;

- une équation de prévision de la digestibilité in vivo à partir de la digestibilité par la pepsine-cellulase qui soit, si possible, la même pour tous les fourrages, ce qui est rarement le cas des méthodes actuellement disponibles et limite donc leur intérêt quand on veut prévoir la digestibilité des fourrages des prairies à flore complexe.

\section{Matériel et méthodes}

\section{Méthode proposée}

Les traitements ont lieu dans des creusets de $90 \mathrm{ml}$ (hauteur $12,5 \mathrm{~cm}$, diamètre intérieur $3 \mathrm{~cm}$ ) munis d'un filtre en verre frité de porosité 2.

Ils comportent les deux étapes décrites par Jones \& HAYwaRd (1975) avec toutefois quelques modifications : 
- un pré-traitement de 24 heures au bain-marie à $40^{\circ}$ avec 0,2 p. 100 de pepsine (MERCK, 7190 pouvoir digestif 1/10 000) dans de l'acide chlorhydrique $1 \mathrm{~N}$ permet une attaque plus facile par la cellulase. On utilise $50 \mathrm{ml}$ de solution pepsine$\mathrm{HCl}$ pour $500 \mathrm{mg}$ d'échantillon broyé à la grille de $0,8 \mathrm{~mm}$;

- après filtration et lavage à l'eau distillée, l'attaque avec la préparation cellulasique s'effectue dans $50 \mathrm{ml}$ de tampon acétate de sodium $0,05 \mathrm{M}$ à $\mathrm{pH} 4,6$, pendant 24 heures au bain-marie à $40^{\circ}$. La préparation cellulasique choisie est extraite de Trichoderma viride : cellulase Onozuka R 10 (Yakult Pharmaceutical Industry Co LTD 21, Shingikancho, Nishinomiya 622 - Japan); elle est employée à raison de $100 \mathrm{mg} / 100 \mathrm{ml}$ de tampon.

Après filtration et rinçage, le résidu est séché à l'étuve à $130^{\circ}$ pendant 48 heures puis pesé. Le résidu représente l'indigestible à la pepsine-cellulase et nous appelons digestibilité par la pepsine-cellulase le pourcentage de matière sèche solubilisé par l'ensemble des deux traitements.

Un fourrage témoin est introduit dans chaque série et permet d'estimer les différences entre les séries.

\section{Fourrages}

Les échantillons utilisés sont représentatifs des principales catégories de fourrages verts, d'ensilages et de foins de graminées, de légumineuses et de prairies naturelles. Leur digestibilité in vivo a été mesurée sur des moutons alimentés ad libitum. Les teneurs en cellulose brute Weende, matières azotées $(\mathrm{N}$ Kjeldahl $\times 6,25)$, cendres, ont été déterminées au préalable.

Douze échantillons (tabl. 2) ont été utilisés pour mettre au point la méthode et 154 pour établir les équations de régression entre la digestibilité in vivo et par la pepsine-cellulase.

Toutes les déterminations ont été effectuées en triple.

\section{Méthode d'analyse statistique}

Les équations de régression entre la digestibilité in vivo de la matière organique et la digestibilité par la pepsine-cellulase ont été établies séparément pour les différents types de fourrages et les différents modes de conservation. Pour obtenir une équation commune à l'ensemble des fourrages, nous ne pouvions utiliser le modèle de régression simple puisque nous n'avions pas le même nombre d'échantillons dans chaque groupe, c'est pourquoi nous avons utilisé le modèle mathématique décrit par SEEBECK (1973) et le programme de calcul sur ordinateur mis au point par cet auteur. 


\section{TABLEAU 2}

Fourrages utilisés pour réaliser les essais méthodologiques (tous sont des échantillons de fourrages verts à l'exception du numéro 11 qui est un foin).

Forages used in these experiments (all forages were analysed as fresh except number 11 as hay).

\begin{tabular}{|c|c|c|c|}
\hline $\begin{array}{l}\text { Variétés } \\
\text { des fourrages utilisés } \\
\text { Forages species }\end{array}$ & $\begin{array}{c}\text { Matières azotées } \\
\text { totales } \\
\text { (p. } 100 \mathrm{MS}) \\
\text { Crude protein } \\
\text { (p. 100 } \\
\text { dry matter) }\end{array}$ & $\begin{array}{l}\text { Cellulose } \\
\text { brute } \\
\text { (p. } 100 \mathrm{MS}) \\
\text { Crude fibre } \\
\text { (p. } 100 \\
\text { dry matter) }\end{array}$ & $\begin{array}{l}\text { Digestibilité } \\
\text { de la matière } \\
\text { organique } \\
\text { Organic matter } \\
\text { digestibility }\end{array}$ \\
\hline $\begin{array}{l}\text { 1. Trèfle violet } \ldots \ldots \ldots \ldots \\
\text { Red clover }\end{array}$ & 16,7 & 20,4 & 0,757 \\
\hline $\begin{array}{l}\text { 2. Trèfle violet } \ldots \ldots \ldots \\
\text { Red clover }\end{array}$ & 13,2 & 26,9 & 0,679 \\
\hline $\begin{array}{l}\text { 3. Trèfle violet } \ldots \ldots \ldots \ldots \\
\text { Red clover }\end{array}$ & 20,9 & 18,1 & 0,762 \\
\hline $\begin{array}{l}\text { 4. Trèfle violet } \ldots \ldots \ldots \ldots \\
\text { Red clover }\end{array}$ & 15,2 & 31,0 & 0,616 \\
\hline $\begin{array}{l}\text { 5. Luzerne } \\
\text { Lucerne }\end{array}$ & 18,0 & 20,2 & 0,725 \\
\hline 6. $\underset{\text { Lucerne }}{\text { Luzerne }} \ldots \ldots \ldots \ldots \ldots$ & 16,8 & 29,4 & 0,645 \\
\hline $\begin{array}{l}\text { 7. Luzerne } \\
\text { Lucerne }\end{array}$ & 23,1 & 23,0 & 0,610 \\
\hline 8. $\underset{\text { Lucerne }}{\text { Luzerne }} \ldots \ldots \cdots \cdots \cdots$ & 18,1 & 29,7 & 0,743 \\
\hline $\begin{array}{l}\text { 9. Ray-grass italien } \ldots \ldots \\
\text { Italian rye-grass }\end{array}$ & 16,2 & 15,9 & 0,805 \\
\hline $\begin{array}{l}\text { 10. Ray-grass italien } \ldots \ldots \\
\text { Italian rye-grass }\end{array}$ & 7,3 & 27,9 & 0,673 \\
\hline $\begin{array}{l}\text { 11. Ray-grass italien } \ldots \ldots \\
\text { Italian rye-grass }\end{array}$ & 9,5 & 23,3 & 0,728 \\
\hline $\begin{array}{l}\text { 12. Ray-grass anglais } \ldots \ldots \\
\text { Perennial rye-grass }\end{array}$ & 11,4 & 28,1 & 0,703 \\
\hline
\end{tabular}




\section{Résultats}

\section{A. Justification de la méthode proposée}

\section{Pré-traitement par la pepsine 0,2 p. 100 dans $\mathrm{HCl}$}

Pour ces essais 7 fourrages seulement ont été utilisés, à savoir les échantillons $1,2,7,8,9,10,11$ du tableau 2 .

\section{a) Concentration en $\mathrm{HCl}$}

Le temps de pré-traitement avec la pepsine 0,2 p. $100-\mathrm{HCl}$ étant fixé à 24 heures, la digestibilité par la pepsine-cellulase a été mesurée pour différentes concentrations en acide : $0,1,0,5,1$ et $2 \mathrm{~N}$ (fig. 1 a). Elle augmente significativement $(p<0,05)$ avec la concentration jusqu'à $1 \mathrm{~N}$ puis elle n'est pas significativement différente entre $1 \mathrm{~N}$ et $2 \mathrm{~N}$. Pour ces différentes concentrations l'écart-type résiduel $\left(\mathrm{S}_{\mathrm{IR}}\right)$ de l'équation liant la digestibilité in vivo à la digestibilité par la pepsinecellulase est respectivement de $0,028,0,027,0,036$ et 0,039 . La précision de la prévision n'est donc pas améliorée lorsque la concentration en acide augmente.

Sur ces mêmes fourrages, et pour une concentration $\mathbf{N}$, nous avons aussi comparé les résultats obtenus avec et sans adjonction de pepsine. La suppression de la pepsine diminue significativement, de 7,0 points, la digestibilité par la pepsine-cellulase ainsi que la liaison avec la digestiblité in vivo $\left(S_{R}=0,041\right.$ sans pepsine contre $S_{R}=0,036$ avec pepsine).

\section{b) Influence de la durée et de la température du pré-traitement} par la pepsine à 0,2 p. 100 dans $\mathrm{HCl}$ (tabl. 3)

Elle a été étudiée sur 12 fourrages présentés au tableau 1. L'allongement de 24 à 48 heures de la durée du pré-traitement n'a pas augmenté significativement la digestibilité par la pepsine-cellulase (respectivement 0,728 et 0,743 ) pas plus que l'élévation de la température de 40 à $50^{\circ}$ (respectivement 0,739 et 0,732 ). Les écartstypes résiduels $S_{\mathrm{R}}$ de la prévision de la digestibilité in vivo à partir de la digestibilité par la pepsine-cellulase n'ont pas été significativement différents selon la durée (les valeurs moyennes sont respectivement de 0,029 et 0,028 pour 24 et 48 heures) et ont été systématiquement un peu plus élevés à $50^{\circ}$ qu'à $40^{\circ}$ (respectivement 0,032 et 0,024 pour les valeurs moyennes).

\section{Traitement par la cellulase}

\section{a) Concentration en cellulase}

L'influence de la concentration en cellulase a été étudiée sur les 7 fourrages précédemment utilisés pour étudier la concentration en acide lors de l'attaque à la pepsine. Le pré-traitement étant celui retenu, soit 0,2 p. 100 de pepsine dans une solution $\mathrm{HCl} 1 \mathrm{~N}$ pendant 24 heures à $40{ }^{\circ} \mathrm{C}$, nous avons comparé 7 concentrations en cellulase, à savoir $0,50,75,100,150,175$ et $200 \mathrm{mg}$ de cellulase $/ 100 \mathrm{ml}$ de tampon. Les résultats obtenus (fig. 1 b) mettent en évidence l'influence très nette 


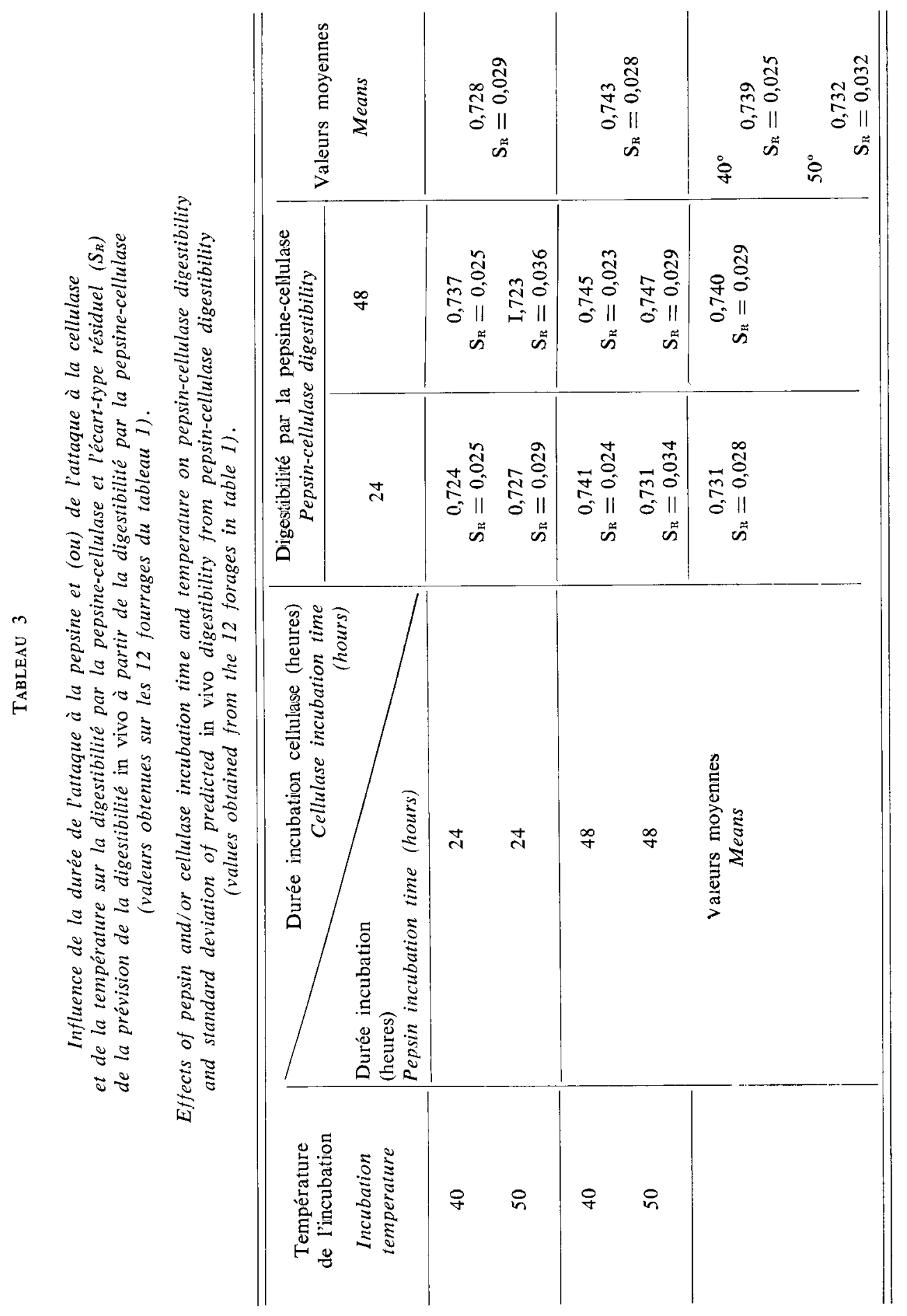


de la présence et de la concentration en enzyme sur la digestibilité par la pepsinecellulase. Celle-ci augmente en moyenne de $0,23(0,21$ à 0,25$)$ entre 0 et $50 \mathrm{mg}$ cellulase $/ 100 \mathrm{ml}$ de tampon puis plus faiblement mais de façon encore significative, entre 50 et $100 \mathrm{mg}$ de cellulase, pour se stabiliser ensuite. L'écart-type résiduel $S_{\mathbf{R}}$ de la prévision de la digestibilité in vivo à partir des digestibilités par la pepsinecellulase est respectivement de $0,031,0,028,0,028,0,027,0,029,0,028,0,028$ pour ces 7 concentrations en cellulase. Les variances sont homogènes ( $\mathrm{X}^{2}$ de BarTLETT).

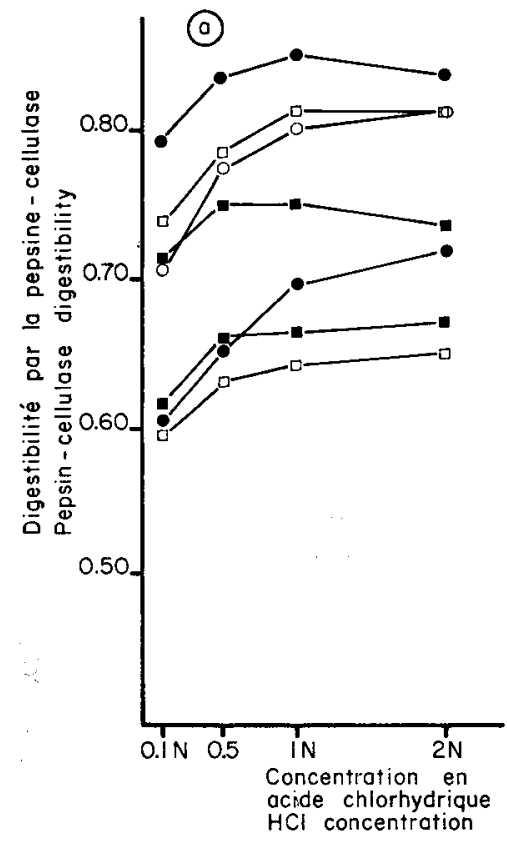

FIG. 1 a

Influence de la concentration en acide.

Effect of acid concentration.

- Fourrage vert de ray-grass italien. Italian rye-grass fresh forage.

- Foin de ray-grass italien. Italian rye-grass hay.

- Fourrage vert de luzerne. Lucerne fresh forage.

- Fourrage vert de trèfle. Red clover fresh forage.

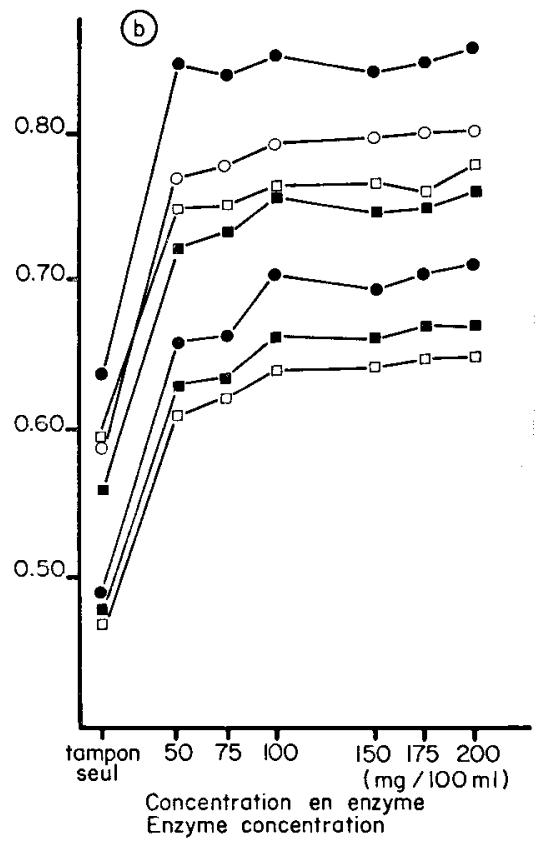

FIG. $1 \mathrm{~b}$

Influence de la concentration en enzyme cellulase «Onozuka $R 10$ ».

Effects of "Onozuka $R 10$ » concentration.

- Fourrage vert de ray-grass italien. Italian rye-grass fresh forage.

- Foin de ray-grass italien. Italian rye-grass hay.

- Fourrage vert de luzerne. Lucerne fresh forage.

- Fourrage vert de trèfle. Red clover fresh forage.

\section{b) Influence de la durée}

Les résultats, rapportés au tableau 3, montrent que l'allongement de la durée d'attaque par la cellulase modifie très peu la digestibilité par la pepsine-cellulase ainsi que l'écart-type résiduel de la prévision de la digestibilité in vivo : respectivement 
en moyenne 0,731 et 0,028 pour 24 heures et 0,740 et 0,029 pour 48 heures. En revanche quand la durée à la fois du pré-traitement par la pepsine $\mathrm{HCl}$ et du traitement par la cellulase augmente de 24 à 48 heures, la digestibilité par la pepsinecellulase augmente significativement $(\mathrm{p}<0,05)$ de 0,727 à 0,754 mais l'écart-type résiduel de la prévision n'est pas amélioré : 0,025 pour $24 \mathrm{~h}-24 \mathrm{~h}$ et $40^{\circ} \mathrm{C}$ contre 0,023 pour $48 \mathrm{~h}-48 \mathrm{~h}$ et $40^{\circ} \mathrm{C}$.

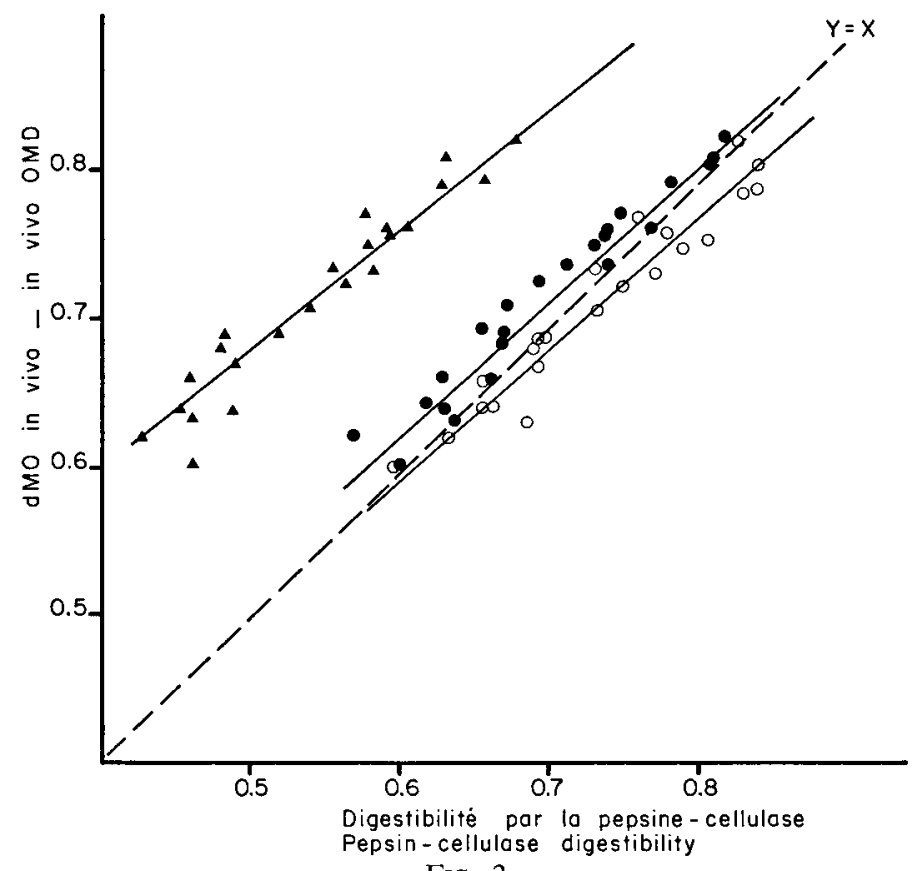

Fig. 2

Relations entre la digestibilité de la matière organique (dMO) in vivo $(Y)$ et la digestibilité par la pepsine-cellulase $(X)$ pour plusievirs variétés de préparations cellulasiques.

Relationships between organic matter in vivo $(Y)$ and pepsin-cellulase digestibility $(X)$ for different enzymes.

○ Cellulase «Onozuka R 10 » $\mathrm{Y}=0,87 \mathrm{X}+0,72 \pm 0,017 \quad \mathrm{r}=0,97 \quad \mathrm{n}=23$. Trichoderma viride.

- Cellulase "Celluclast» Trichoderma viride.

- Cellulase «Sigma» Aspergillus niger. - . - $\mathrm{Y}=\mathrm{X}$.

$$
\begin{array}{lll}
\mathrm{Y}=0,86 \mathrm{X}+0,10 \pm 0,014 & \mathrm{r}=0,98 & \mathrm{n}=23 . \\
\mathrm{Y}=0,84 \mathrm{X}+0,26 \pm 0,017 & \mathrm{r}=0,96 & \mathrm{n}=23 .
\end{array}
$$

c) Comparaison entre plusieurs types de préparations cellulasiques

Nous avons comparé sur 23 échantilons de fourrages verts de graminées l'activité de deux préparations cellulasiques extraites de Trichoderma viride, cellulase "Onozuka R 10 » et «celluclast 2505 type $\mathrm{N}$ », ainsi que d'une cellulase «Sigma » extraite d'Aspergillus niger. Les droites de régression entre la digestibilité in vivo et celle 
par la pepsine-cellulase pour les trois types d'enzymes sont présentées à la figure 2 . $\mathrm{Ce}$ sont des droites pratiquement parallèles mais l'ordonnée à l'origine est différente. C'est avec la cellulase «Onozuka $\mathbf{R} 10$ » que la digestibilité par la cellulase est la plus proche de la digestibilité in vivo. La droite de régression est proche de la bissectrice $(\mathrm{Y}=\mathrm{X})$. Avec la cellulase «Celluclast» et la cellulase «Sigma» le pourcentage de matière sèche solubilisé est moins important. La précision est très bonne dans tous les cas, $r$ et $S_{\mathrm{R}}$ étant compris respectivement entre 0,96 et 0,98 et entre 0,013 et 0,017 .

\section{Répétabilité et reproductibilité}

La répétabilité intra série (r) et la reproductibilité entre séries (R) de la méthode proposée ont été calculées à partir des déterminations (en triple) de la digestibilité par la pepsine-cellulase du foin témoin qui a été inclus dans chaque série. $r$ et $R$ représentent l'écart arithmétique entre deux déterminations de la digestibilité par la pepsine-cellulase d'un échantillon ayant une probabilité de 95 p. 100 de ne pas être dépassé (Grappin, 1976; SNedecor \& Cochran, 1971).

Ces valeurs peuvent être estimées à partir des formules suivantes :

$$
r=\frac{t}{\sqrt{n}} \times \sigma r \quad \text { et } \quad R=\frac{t}{\sqrt{n}} \times \sigma R
$$

$t$ est le $\mathrm{t}$ de Student à la probabilité 0,05 ,

$\mathrm{n}=$ le nombre de répétitions,

$\sigma^{2} \mathrm{r}=$ variance résiduelle calculée à partir d'une analyse de variance à une voie de classification,

$\sigma^{2} \mathrm{R}=\sigma^{2} \mathrm{r}+\sigma^{2} \mathrm{~L}$ (avec $\sigma^{2} \mathrm{~L}$ : variance correspondant à l'effet série).

Les valeurs de $r$ et $R$ sont respectivement de 0,004 et 0,008 , ce qui montre que la méthode proposée est à la fois très répétable et très reproductible. La détermination de la digestibilité par la pepsine-cellulase peut donc se faire à la rigueur en simple et la présence dans chaque série d'un échantillon témoin n'est pas obligatoire, bien que souhaitable.

\section{B. Application de cette méthode pour prévoir la digestibilité par la pepsine-cellulase}

La méthode mise au point a été appliquée à 154 échantillons de fourrages verts et conservés, de légumineuses et de prairies naturelles. Les équations de régression liant la digestibilité in vivo de la matière organique à la digestibilité par la pepsine-cellulase sont données au tableau 4 pour les différentes catégories de fourrages. En ce qui concerne les ensilages, la digestibilité in vivo retenue est la digestibilité non corrigée pour les pertes de matières volatiles lors de la détermination de la teneur en matière sèche de l'ensilage par séchage à l'étuve, puisque l'échantillon utilisé pour la détermination de la digestibilité par la pepsine-cellulase est un échantillon sec. 


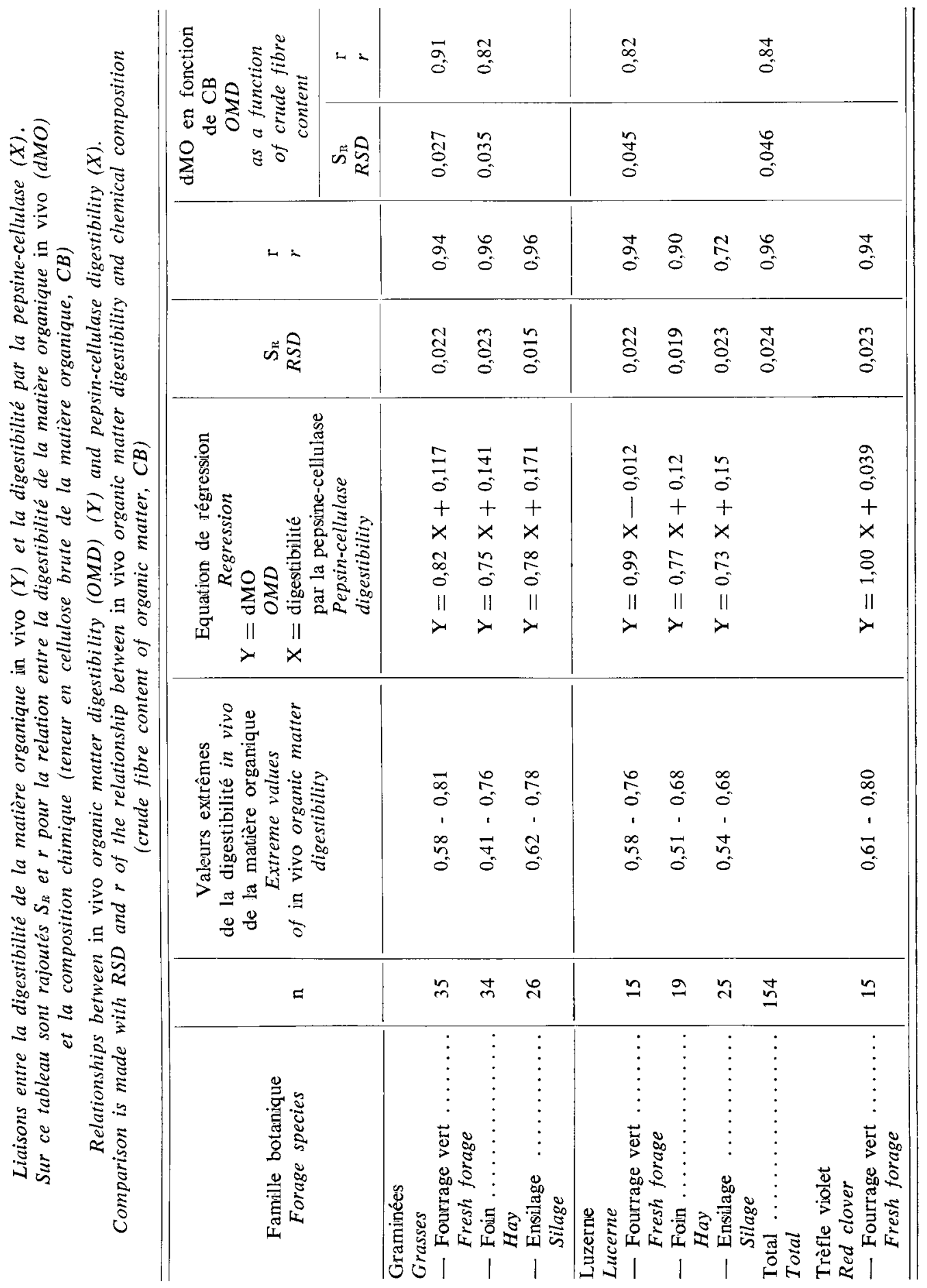


Pour ces mêmes échantillons, la méthode par la pepsine-cellulase permet une bien meilleure prévision de la digestibilité in vivo $\left(S_{\mathrm{R}}\right.$ compris entre 0,015 et 0,023 ) que la cellulose brute $\left(S_{12}\right.$ compris entre 0,027 et 0,046 ) (la prise en compte de la teneur en matières azotées en plus de la teneur en cellulose brute n'améliore pas la précision de la prévision par comparaison à la cellulose brute seule). D'ailleurs en regroupant l'ensemble des 154 fourrages à l'exception des trèfles violets, $S_{\mathrm{R}}$ est respectivement égal à 0,024 et 0,046 . Les trèfles violets n'ont pas été inclus parce qu'à même digestibilité in vivo que les autres fourrages, leur digestibilité par la pepsine-cellulase est en moyenne inférieure de 0,057 unité.

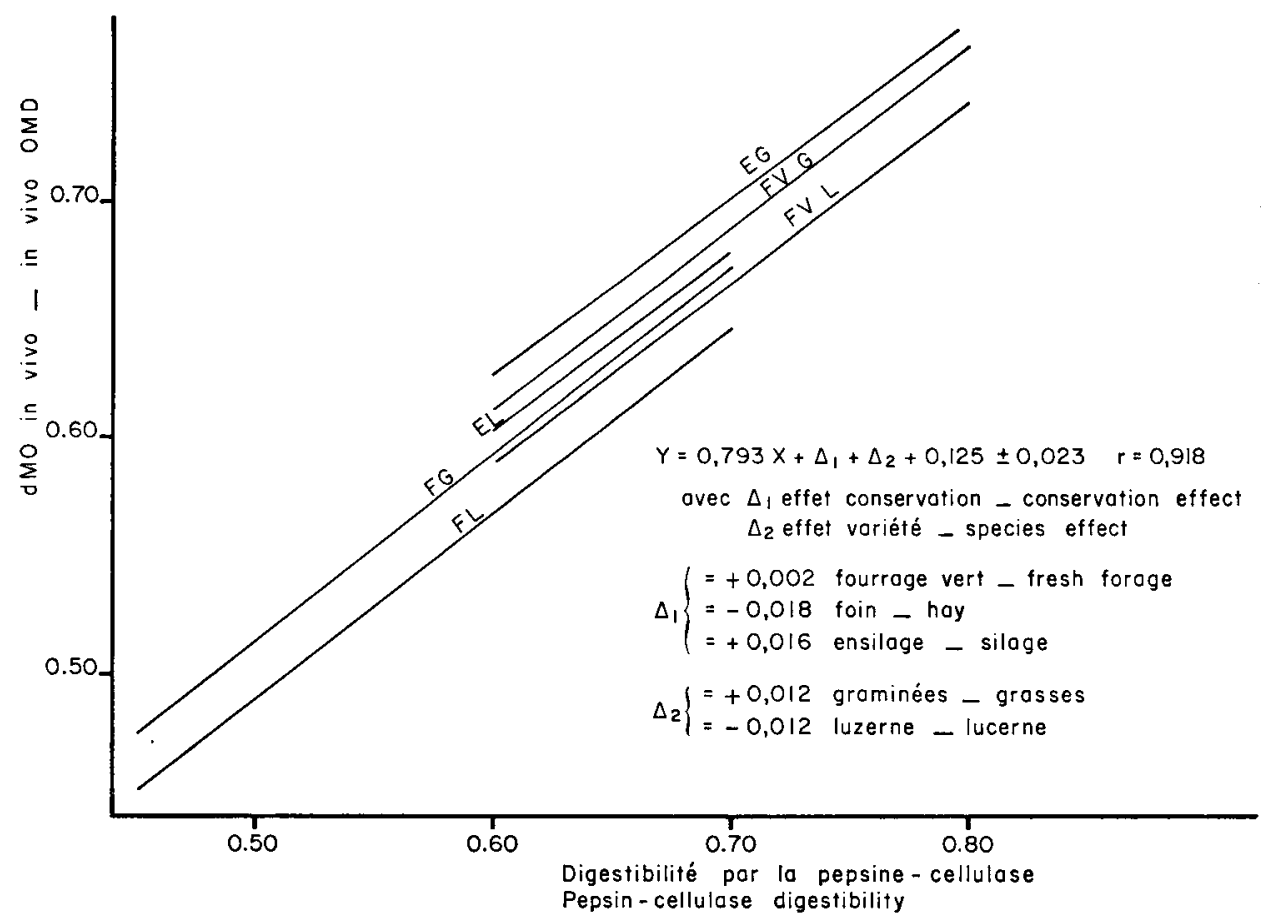

FiG. 3

Relations entre la digestibilité in vivo de la matière organique (dMO) et la digestibilité par la pepsine-cellulase.

Ces résultats sont obtenus à l'ordinateur par le programme Seebeck pour différents types de fourrages.

Relationship between in vivo organic matter digestibility and pepsin-cellulase digestibility.

The results were obtained with a Seebeck computer programme for different forages.

FVG : Fourrage vert de graminées. Grasses fresh forage.

FVL : Fourrage vert de luzerne. Lucerne fresh forage.

FG : Foin de graminées. Grasses hay.
FL : Foin de luzerne. Lucerne hay.

EG : Ensilage de graminées. Grasses silage.

EL : Ensilage de luzerne.

Lucerne silage. 
Les pentes des équations de régression liant la digestibilité in vivo (Y) à la digestibilité par la pepsine-cellulase (X) n'étant pas significativement différentes, il est possible de proposer une équation de régression unique grâce au programme mis au point par SeEBeck (1973) (figure 3). Celle-ci est la suivante pour les 154 échantillons de fourrages autres que le trèfle violet :

$$
\mathrm{Y}=0,793 \mathrm{X}+0,125+\Delta_{1}+\Delta_{2} \pm 0,023 \quad \mathrm{r}=0,918 \quad \mathrm{n}=154
$$

avec $\Delta_{1}=+0,002$ pour les fourrages verts,

$-0,018$ pour les foins,

$+0,016$ pour les ensilages,

et $\Delta_{2}=+0,012$ pour les graminées,

$-0,012$ pour les luzernes.

Si on ne prend pas en compte les ensilages, dont il vaut mieux prévoir la digestibilité à partir de celle du fourrage vert à la mise en silo (Michalet-Doreau \& Demarquilly, 1981) l'équation devient la suivante :

$$
\begin{aligned}
\mathrm{Y}= & 0,79 \mathrm{X}+0,123+\Delta \pm 0,022 \quad \mathrm{r}=0,967 \quad \mathrm{n}=103 \\
\text { avec } \Delta= & +0,013 \text { pour les fourrages verts de graminées, } \\
& +0,013 \text { pour les fourrages verts de luzerne, } \\
& -0,008 \text { pour les foins de graminées, } \\
& -0,019 \text { pour les foins de luzerne. }
\end{aligned}
$$

On constate qu'à même digestibilité par la pepsine-cellulase, la digestibilité in vivo est un peu plus élevée pour les fourrages verts que pour les foins et pour les graminées que pour les luzernes. Les différences restent cependant très faibles puisque les valeurs de $\Delta$ restent comprises dans l'équation (1) entre $+0,016$ et $-0,018$ et dans l'équation (2) entre $+0,013$ et $-0,019$, de sorte que ces équations, notamment la seconde, peuvent être utilisées sans leur $\Delta$ pour prévoir la digestibilité des fourrages quels que soient leur famille botanique ou leur mode de conservation à l'exception des fourrages à base de trèfle violet.

En revanche, l'équation à appliquer aux trèfles violets est la suivante :

$$
\mathrm{Y}=1,05 \mathrm{X}+0,017 \pm 0,030 \quad \mathrm{r}=0,89 \quad \mathrm{n}=15
$$

Or on ne sait pas si, comme pour les autres fourrages, il existe de petites différences entre les fourrages verts et les foins puisque cette relation n'a été établie que pour Ies fourrages verts.

\section{Discussion}

Nos résultats, en accord avec ceux de Jones \& HaYward (1975) et de Goto \& Minson (1977), montrent que le pré-traitement par la pepsine- $\mathrm{HCl}$ augmente la matière sèche qui est ensuite solubilisée par la cellulase et surtout améliore la 
prévision de la digestibilité in vivo. La concentration en acide que nous préconisons (1 N) est cependant beaucoup plus élevée que celle utilisée par les autres auteurs : 0,1 N (Jones \& Hayward, 1975), 0,075 N (Adegbola \& Paladines, 1977), $0,1 \mathrm{~N}$ à $0,125 \mathrm{~N}$ (Allison \& BorzUCKI, 1978), mais plus faible que celle utilisée par Kellner \& Kirchgessner (1977), $2 \mathrm{~N}$ à $100^{\circ}$ pendant 30 minutes. Il est probable qu'à cette concentration $(1 \mathrm{~N}), \mathrm{HCl}$ dissout une partie des hémicelluloses (Salo, 1957 ; JARrige, 1961 ; VAN Soest, 1967). Nous avons adopté cette concentration parce qu'elle permet d'obtenir des digestibilités par la pepsine-cellulase voisines de la digestibilité in vivo, bien que la précision de la prévision soit pratiquement indépendante de la concentration en acide entre 0,1 et $1 \mathrm{~N}$.

En revanche, si la durée du pré-traitement $\mathrm{HCl}$-pepsine passe de 24 à 48 heures, la digestibilité par la pepsine-cellulase n'augmente que très peu et sa liaison avec la digestibilité in vivo n'est pas améliorée. Notre but étant d'obtenir une méthode rapide et précise, notre choix s'est done porté sur une durée de 24 heures.

De nombreuses cellulases existent actuellement dans le commerce. Elles ne sont d'ailleurs jamais pures, car toujours contaminées par d'autres enzymes telles que les hémicellulases. Celle que nous préconisons, la cellulase "Onozuka R 10 » est extraite de Trichoderma viride. Elle est particulièrement active et on peut l'employer à des doses $(50 \mathrm{mg} / 500 \mathrm{mg}$ de fourrage) beaucoup plus faibles que celles préconisées par les autres auteurs et qui varient de $125 \mathrm{mg}$ d' "Onozuka SS P 1500 » (GoTo \& Minson, 1977) à $900 \mathrm{mg}$ (KIRCHGESSNER \& KELLNER, 1978) toujours pour $500 \mathrm{mg}$ de fourrage, mais cela est peut-être aussi le résultat de la concentration $1 \mathrm{~N}$ adopté pour le pré-traitement par la pepsine-cellulase. Cet aspect est intéressant compte tenu du prix relativement élevé des cellulases. La cellulase «Onozuka R 10 » permet d'obtenir une digestibilité par la pepsine-cellulase du même ordre de grandeur, en moyenne, que la digestibilité in vivo mais la précision de la prévision de la digestibilité in vivo n'est pas meilleure que celle permise par les deux autres cellulases «Celluclast » et «Sigma » testées.

L'allongement de 24 à 48 heures de la durée d'attaque par la cellulase, n'augmente que très faiblement la matière sèche solubilisée. Certes, de nombreux auteurs ont vérifié que la digestion par les cellulases est rapide mais les résultats de REXEN (1977) montrent bien que cette rapidité dépend du type de cellulase employée. D'un point de vue pratique il est intéressant de pouvoir réduire à 24 heures le temps d'attaque tant par la pepsine que par la cellulase et cela sans diminuer la précision de la prévision de la digestibilité in vivo, contrairement à ce qui a été trouvé par Mc LEOD \& Minson (1978).

Nos résultats, en accord avec ceux de Jones \& Hayward (1973), Rexen (1977), montrent que l'augmentation de 40 à $50^{\circ} \mathrm{C}$ de la température d'incubation n'a pas d'effet sur la digestibilité par la pepsine-cellulase contrairement aux résultats de MC Leod \& Minson (1978), Allison \& BorzucKi (1978) et qu'à $50^{\circ} \mathrm{C}$ la précision de la prévision de la digestibilité in vivo diminue. Tous les auteurs, sauf Roughan \& Holland (1977) préconisent d'ailleurs de travailler à $40^{\circ} \mathrm{C}$.

Nous ne sommes pas arrivés, comme Kellner \& Kirchgessner (1977) à trouver une digestibilité par la pepsine-cellulase pratiquement identique à la digestibilité in vivo mais leur traitement «cellulasique» comporte trois opérations (un prétraitement $\mathrm{HCl} 2 \mathrm{~N}$, un traitement à la cellulase, un post-traitement $\mathrm{HCl}$-pepsine) ce qui alourdit considérablement la méthode et lui enlève de son intérêt. Cependant 
leur méthode sous-estıme un peu la digestibilité quand celle-ci est faible, de sorte que le recours à une équation de régression liant les digestibilités in vivo et par la pepsine-cellulase est, pour elle aussi, nécessaire.

Comme d'autres auteurs l'ont montré avant nous (cf. tabl. 1) nos résultats indiquent que la digestibilité par la pepsine-cellulase permet de prévoir la digestibilité in vivo des fourrages de façon précise et avec de bonnes reproductibilité et répétabilité. Si on excepte les trèfles violets, il est possible de proposer une seule équation quelles que soient la famille et la nature (vert ou foin) du fourrage. Il est souhaitable cependant de tenir compte de la nature du fourrage et dans une moindre mesure de sa famille.

A même digestibilité in vivo la matière sèche solubilisée par la pepsine-cellulase est plus faible pour les fourrages verts que pour les foins et pour les graminées que pour les légumineuses. Cela doit résulter du fait que la pepsine-cellulase dissout moins de constituants pariétaux que les enzymes ou les microbes du rumen et du tube digestif du ruminant. Ce résultat avait été remarqué également par MC QueEN \& VAN SoEst (1975). On sait en effet qu'à même digestibilité in vivo les graminées sont plus riches en constituants pariétaux que les légumineuses et les fourrages verts plus riches que les foins. Or la pepsine-cellulase solubilise moins ces constituants pariétaux que le ruminant et il en résulte qu'à même digestibilité in vivo, la digestibilité par la pepsine-cellulase est plus faible pour les graminées que pour les légumineuses et est également plus faible pour les fourrages verts que pour les foins. JARRIgE \& THIVEND (1969) avaient également trouvé que les fourrages verts étaient moins solubilisés que les foins. De même Mc LEOD \& Minson (1978) remarquent que la cellulase solubilise moins les graminées que les légumineuses de même digestibilité in vivo mais proposent tout de même une seule droite de régression tandis que Terry, Mundell \& Osbourn (1978) proposent une équation différente pour les graminées et les légumineuses.

Avec les trèfles violets, nous avons obtenu une proportion de matière sèche solubilisée par la cellulase plus faible que pour l'ensemble des autres fourrages (graminées et légumineuses). Cette différence semble difficile à expliquer car elle n'a pas été signalée par Jones \& HaYward (1975) dont 12 des 25 échantillons de légumineuses, utilisés pour établir la liaison entre les digestibilités in vitro et par la pepsinecellulase, étaient des trèfles violets. Peut-être est-ce dû au fait que nos échantillons de trèfle étaient anciens (ils avaient plus de 10 ans) ce qui n'était pas le cas pour les autres échantillons.

En définitive la méthode de digestibilité par la pepsine-cellulase proposée permet, à partir d'une seule équation de régression, de prévoir la digestibilité avec une précision équivalente à celle permise par la composition chimique mais en utilisant alors des équations spécifiques à chaque espèce, numéro de cycle de végétation et nature (fourrage vert, foin, ensilage) du fourrage (cf. Andrieu, Demarquilly \& WeGat-Litre, 1981). Elle peut donc rendre de grands services pour prévoir la digestibilité des fourrages des prairies à flore complexe d'autant qu'elle est, contrairement aux méthodes de digestibilité in vitro, facile à mettre en œuvre dans les laboratoires d'analyses en série puisqu'elle ne nécessite pas l'entretien d'animaux fistulisés donneurs de jus de rumen. Ses principaux avantages par rapport aux autres méthodes à pepsine-cellulase proposées ces dernières années sont de cumuler les avantages suivants :

- la rapidité : 2 traitements de 24 heures chacun, alors que les autres mé- 
thodes comportent le plus souvent deux traitements de 48 heures chacun ou l'un de 24 et l'autre de 48 heures;

- l'utilisation d'une équation de régression unique;

- la facilité de mise en cuvre puisque toutes les opérations se font dans le même creuset filtrant, ce qui évite les transvasements et les récupérations des résidus sur un filtre ou les centrifugations. Elle explique en grande partie les très bonnes répétabilité et reproductibilité de la méthode.

Accepté pour publication en avril 1982.

\section{Summary}

\section{Prediction of forage digestibility by means of an enzymatic procedure}

A modification of the JoNES \& HAYWARD (1975) method for prediction of in vivo digestibility of forages is described. Ground dried forages $(0.5 \mathrm{~g})$ were first digested in $50 \mathrm{ml}$ of a pepsin solution in $1 \mathrm{~N}$ chlorhydric acid at $40^{\circ}$ for $24 \mathrm{~h}$. The samples were then further solubilised in $50 \mathrm{ml} 0.05 \mathrm{M}$ acetate buffer solution containing $0.1 \mathrm{p} .100(\mathrm{~W} / \mathrm{V})$ "Onozuka R 10 » cellulase at $40^{\circ}$ for $24 \mathrm{~h}$.

The correlation between in vivo organic matter digestibility and pepsin-cellulase digestibility were markedly improved by pre-treatment of herbage with acid-pepsin (RSD $=0.036$ with pepsin, $\operatorname{RSD}=0.041$ without pepsin), but no significant effect could be found of acid concentration from 0.1 to $1 \mathrm{~N}$ in pepsin solution (figure 1 a), though values obtained at $1 \mathrm{~N}$ concentration were closer to those obtained in vivo. The accuracy of the prediction by the pepsin-cellulase method was not improved when temperature was increased from $40^{\circ}$ to $50^{\circ}$ or when incubation time was $48 \mathrm{~h}$ instead of $24 \mathrm{~h}$ (table 3).

Pepsin-cellulase digestibility of forages was slightly but significantly increased when cellulase concentration was changed from 50 to $100 \mathrm{mg} / 100 \mathrm{ml}$ of a buffer solution, but was not further increased by concentrations ranging from 100 to $200 \mathrm{mg} / 100 \mathrm{ml}$ buffer solution (fig. $1 \mathrm{~b}$ ).

However, the accuracy of the in vivo digestibility prediction by the pepsin-cellulase method was similar at any of these six concentrations. A comparison of three different cellulases ( Onozuka R $10 »$, "celluclast», and «Sigma ») did not show any differences of accuracy between these cellulases ( $R S D=0.017,0.013,0.017$, respectively) (figure 2), though values provided by the method using «Onozuka $\mathrm{R} 10$ » were close to the in vivo digestibility coefficients.

We therefore used this «Onozuka $\mathbf{R} 10$ » preparation to establish a relationship between dry matter digestibility by pepsin-cellulase solution $(\mathrm{X})$ and in vivo organic matter digestibility (Y).

An equation established from 154 samples of fresh forages, hays and silages of grasses and leguminous plants (apart red clover) showed that the pepsin-cellulase method was more accurate than the method based on chemical analysis to predict forage digestibility.

$$
\mathrm{Y}=0.793 \mathrm{X}+0.125+\Delta_{1}+\Delta_{2} \quad \mathrm{RSD}=0.023 \quad \mathrm{r}=0.918
$$

with $\Delta_{1}=+0.002$ fresh forage

$$
\begin{aligned}
\Delta_{1}= & +0.002 \text { fresh for } \\
& +0.018 \text { hay } \\
& +0.016 \text { silage } \\
\Delta_{2}= & +0.012 \text { grasses } \\
& -0.012 \text { lucerne. }
\end{aligned}
$$




\section{Références bibliographiques}

Abe A., Hori S., Kameoka K., 1972. Development and application of cellulase hydrolysis for predicting digestibility of roughage. 2 - Influence of pretreatment of samples on cellulase hydrolysis. Jpn. J. zoot. Sci., 43, 146-154.

Adamson A.H., Terry G.R., 1980. The relationship between the in vivo digestibility of hay and its solubility in pepsin-hydrochloric acid and fungal cellulase solutions. $J$. Sci. Food Agric., 31, 854-856.

Adegbola A.A., Paladines O., 1977. Prediction of the digestibility of the dry matter of tropical forages from their solubility in fungal cellulase solutions. J. Sci. Food. Agric., 28, 775-785.

Allison M., BorzucKi R., 1978. Cellulase methods for the efficient digestion of grasses and brassicas. J. Sci. Food Agric., 29, 293-297.

Andrieu J., Demarquilly C., Wegat-Litre E., 1981. Tables de prévision de la valeur alimentaire des fourrages. XI ${ }^{\text {es }}$ Journées du Grenier de Theix, in : Prévision de la valeur nutritive des aliments des ruminants, Ed. I.N.R.A. Publications, 343-591.

Carler L.A., van Hee L.P., Andries A.P., 1979. Estimation de la digestibilité des fourrages grossiers par le traitement à la pepsine-cellulase ou au jus de rumen-pepsine. Rev. Agric., 1, 32, 147-157.

Clark J., Beard J., 1977. Prediction of the digestibility of ruminant feeds from their solubility in enzyme solutions. Anim. Feed. Sci. Technol., 2, 153-159.

Demarquilly C., Weiss Ph., 1970. Tableaux de la valeur alimentaire des fourrages. I.N.R.A., Etude S.E.I., $\mathrm{n}^{\circ} 42$, pp. 1-65.

Demarquilly C., Andrieu J., Sauvant D., 1978. Tableaux de la valeur nutritive des aliments. Chap. 17, in : L'alimentation des ruminants, I.N.R.A., 1978. Ed. I.N.R.A. Publications, Route de Saint-Cyr, 78000 Versailles.

Demarquilly C., Jarrige R., 1981. Panorama des méthodes de prévision de la digestibilité et de la valeur énergétique des fourrages. $\mathrm{XI}^{\mathrm{es}}$ Journées du Grenier de Theix, in : Prévision de la valeur nutritive des aliments des ruminants, Ed. I.N.R.A. Publications, 41-59.

Donefer E., Niemann P.J., Crampton E.W., Llyod L.E., 1963. Dry matter disappearance by enzyme and aqueous solutions to predict the nutritive value of forages. J. Dairy Sci., 46, 965-970.

Dowman M.G., Collins F.C., 1977. The prediction of the digestibility of silage using cellulase. J. Sci. Food Agric., 28, 1071-1074.

Goto I., Minson D.J., 1977. Prediction of the dry matter digestibility of tropical grasses using a pepsin-cellulase assay. Anim. Feed. Sci. Technol., 2, 247-253.

Grappin, 1976. Guide pour l'évaluation des méthodes d'analyses de routine. Le Lait, $555-560,605-621$.

Guggolz J., Saunders R.M., Kohler G.O. et Klopfenstein T.J., 1971. Enzymatic evaluation of processes for improving agricultural wastes for ruminant feeds. J. Anim. $S c i$., 33, 167-170.

Hartley R.D., Jones E.L., Fenlon J.S., 1974. Prediction of the digestibility of forages by treatment of their cell walls with cellulolytic enzymes. J. Sci. Food Agric., 25, 947-954.

Israelsen M., Rexen B., Vestergaard Thomsen K., 1978. Cellulase insoluble fibre as a measure of unvailable organic matter in cattle compounds containing alkali-treated straw. Anim. Feed. Sci. Technol., 3, 227-234.

JARRIGE R., 1961. Analyse des constituants glucidiques des plantes fourragères. Ann. Biol. anim. Bioch. Biophys., 1, 163-202.

Jarrige R., Thivend P., 1969. Action d'une cellulase fongique sur les membranes et son intérêt pour prévoir la digestibilité des plantes fourragères. Ann. Biol. anim. Biochim. Biophys., 9, 171-190. 
Jarrige R., Thivend P., Demarquilly C., 1970. Development of a cellulolytic enzyme digestion for predicting the nutritive value of forages. In : Proc, 11th Intern. Grassl. Congr., Paradise, Australia, 762-768.

Jones D.I.H., Hayward M.V., 1973. A cellulase digestion technique for predicting the dry matter digestibility of grasses. J. Sci. Food Agric., 24, 1419-1426.

Jones D.I.H., Hayward M.V., 1975. The effect of pepsin pretreatment of herbage on the prediction of dry matter digestibility from solubility in fungal cellulase solutions. J. Sci. Food Agric., 26, 711-718.

Kellner R.J., Kirchgessner M., 1977. Estimation of forage digestibility by a cellulase method. Z. Tierphysiol. Tierernährg. Futtermittelkde, 39, 9-16.

KIRChGesSner M., Kellner R.J., 1978. Estimation of digestibility, metabolizable energy and net energy of forage by a cellulase method. Livest. Prod. Sci., 5, 373-377.

MC LEOD M.N., MINSON D.J., 1978. The accuracy of the pepsin-cellulase technique for estimating the dry matter digestibility in vivo of grasses and legumes. Anim. Feed. Sci. Technol., 3, 277-287.

MC LEOD M.N., Minson D.J., 1980. A note on Onozuka 35 cellulase as a replacement for Onozuka SS (P 1500) cellulase when estimating forage digestibility in vitro. Anim. Feed. Sci. Technol., 5, 347-350.

Mc Queen R., Van Soest P.J., 1975. Fungal cellulase and hemicellulase prediction of forage digestibility. J. Dairy Sci., 58, 1482-1491.

Michalet-Doreau B., Demarquilly C., 1981. Prévision de la valeur énergétique des ensilages d'herbe. $\mathrm{XI}^{\mathrm{es}}$ Journées du Grenier de Theix. In : Prévision de la valeur nutritive des aliments des ruminants. Ed. I.N.R.A. Publications, 105-117.

ReXen B., 1977. Enzyme solubility. A method for evaluating the digestibility of alkalitreated straw. Anim. Feed. Sci. Technol., 2, 205-218.

Roughan P.G., Holland R., 1977. Predicting in vivo digestibilities of herbages by exhaustive enzymic hydrolysis of cell walls. J. Sci. Food Agric., 28, 1057-1064.

Salo M.L., 1957. Lignin studies. I - Investigation concerning lignin determinations. Maataloust. Aikakausk, 29, 185-193.

SeEbECK R.M., 1973. The effect of body weight loss on the composition of Braham cross and Africanded cross steers. I - Empty body weight, dressed carcass weight, and offal components. J. agric. Sci., 80, 201-210.

Snedecor G.W., Cochran W.G., 1971. Méthodes statistiques. Ed. Association de Coordination technique agricole, 149, rue de Bercy, 75012 Paris, $6^{\circ}$ édition, 287-533.

Terry R.A., Mundell D.C., Osbourn D.F., 1978. Comparison of two in vitro procedures using rumen liquor-pepsine or pepsin-cellulase for prediction of forage digestibility. J. br. Grassl. Soc., 33, 13-18.

Tinnimit P., Thomas J.W., 1976. Forage evaluation using various laboratory techniques. J. anim. Sci., 43, 5, 1051-1065.

VAN SOEST P.J., 1967. Development of a comprehensive system of feed analyses and its application to forages. J. anim. Sci., 28, 119-128. 